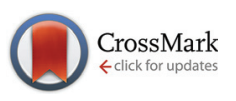

Cite this: Dalton Trans., 2016, 45, 3380

Received 7th December 2015, Accepted 13th January 2016

DOI: $10.1039 / c 5 d t 04772 b$

www.rsc.org/dalton

\title{
Optical contrast and cycling of bistable luminescence properties in $\mathrm{Rb}_{2} \mathrm{KIn}_{(1-x)} \mathrm{Ce}_{x} \mathrm{~F}_{6}$ compounds $\uparrow$
}

\author{
Lucile Cornu, ${ }^{a, b}$ Manuel Gaudon, ${ }^{a, b}$ Olivier Toulemonde, ${ }^{a, b}$ Philippe Veber $^{a, b}$ and \\ Véronique Jubera*a,b
}

\begin{abstract}
Irradiation cycling was performed to evaluate the ageing of the redox process observed on cerium-doped $\mathrm{Rb}_{2} \mathrm{KInF}_{6}$ compounds. An on-off switch of monovalent indium luminescence is observed for the lowest cerium doped material, whereas a nice colourimetric contrast between a bluish-green and orange emission is generated for the material with the highest cerium content. Photoluminescent properties combined with $\mathrm{X}$-ray diffraction, chemical analysis and magnetism measurements allowed an accurate characterization of the system. A complete explanation of the optical behaviour is therefore proposed. Finally, printing tests were performed to illustrate the good functionality of the prepared materials for UV sensitivity.
\end{abstract}

\section{Introduction}

The elpasolite-type materials are widely studied, ${ }^{1}$ and used in various areas such as laser devices, ${ }^{2,3}$ quantum cutters, ${ }^{4}$ neutron and gamma detectors, ${ }^{5}$ and light-emitting diodes. ${ }^{6}$ Applications are also found for data storage and medical radiation detection devices. ${ }^{7,8}$ These matrices of general formula $\mathrm{A}_{2} \mathrm{BB}^{\prime} \mathrm{X}_{6}$ offer a wide variety of compositions: chloride, bromide and fluoride, with their various associated properties. $^{9-12} \mathrm{~A}$ and $\mathrm{B}$ elements belong to the alkali group, B' is a transition metal and $\mathrm{X}$ is a halogen. ${ }^{13}$ At room temperature, the elpasolite-type structure crystallizes in the $F m \overline{3} m$ space group and exhibits two types of metal sites with octahedral and cuboctahedron geometry. ${ }^{14}$ The $\mathrm{B}$ and $\mathrm{B}^{\prime}$ cations are located at the $4 \mathrm{~b}$ and $4 \mathrm{~b}$ Wyckoff positions and $\mathrm{A}$ in the $8 \mathrm{c}$ position. In one previous study on $\mathrm{Rb}_{2} \mathrm{KInF}_{6}: \mathrm{Ce}^{3+}$ compounds, halogen anions were assigned to occupy the $24 \mathrm{e}$ position, ${ }^{14}$ but we have demonstrated that the fluorine ions can be distributed around this average position, e.g., occupying the four sub-positions $96 \mathrm{j}$ with $1 / 4$ occupancy preferentially in a random way. ${ }^{15}$ These considerations provide the understanding of the stabilization process of the structure despite a tolerance factor significantly below 1. Actually, octahedral tilts generate a relaxation of the steric constraints because the $\mathrm{B}$ and $\mathrm{B}^{\prime}$ cations are much too big in comparison to the A cation.

\footnotetext{
${ }^{a}$ CNRS, ICMCB, UPR 9048, F-33600 Pessac, France

${ }^{b}$ Univ. Bordeaux, ICMCB, UPR 9048, F-33600 Pessac, France.

E-mail: veronique.jubera@u-bordeaux.fr, manuel.gaudon@u-bordeaux.fr

$\dagger$ Electronic supplementary information (ESI) available. See DOI: 10.1039/ c5dt04772b
}

A better fit of the experimental cation valences to the theoretical values is therefore obtained. In addition, for an elpasolite matrix doped with optically active cations, optical properties are closely linked with the luminescent cation distribution in the different sites of the structure. Various emission wavelengths can be obtained as demonstrated in a previous study. ${ }^{7,15}$ These materials also show photo-chromo-luminescent properties, resulting in a variation of emission wavelengths after UV radiation exposure. These results reflect the variation of the oxidation state of the cerium and indium cations, according to the equation $2 \mathrm{Ce}^{3+}+\mathrm{In}^{3+} \leftrightarrow 2 \mathrm{Ce}^{4+}+\mathrm{In}^{+}$. Depending on the irradiation wavelength, the redox reaction can occur in the direct or the reverse sense; the emission wavelength variation is due to the optical properties of $\mathrm{Ce}^{3+}$ and $\mathrm{In}^{+}$ luminescence characteristics. An $\mathrm{In}^{+}$orange-red emission appears after a few minutes under UV exposure, linked with the increase of the monovalent indium concentration. As this redox phenomenon is reversible, the $\mathrm{In}^{+}$emission can be switched off using an irradiation at a higher energy. Here, the impact of the synthesis protocol and the percentage of cerium introduced on the photochromism will be discussed. First, the luminescent properties of $\mathrm{Rb}_{2} \mathrm{KIn}_{(1-x)} \mathrm{Ce}_{x} \mathrm{~F}_{6}$ elpasolite compounds with different "target" $\mathrm{Ce}^{3+}$ concentrations $(x=0.02$ and 0.10 ) are investigated. The concentration of the trivalent cerium actually introduced into our matrixes is then determined using coupling X-ray diffraction, ICP spectroscopy and magnetic measurements. New optical properties are observed and fully discussed with regard to the X-ray diffraction and the magnetic analyses. Finally, the potentiality of the compounds to be used as photosensors is evaluated from cyclability tests on the powder and printing tests on monocrystals. 


\section{Experimental section}

Materials and methods

Polycrystalline powder synthesis. To obtain a polycrystalline powder, an aqueous solution process was used. Two solutions were prepared, indium oxide (Alfa Aesar, 99.997\%) was dissolved in hydrofluoric acid (40 mol\% in water), and rubidium carbonate (Alfa Aesar, 99\%), potassium carbonate (Sigma, $>\mathbf{9 9 . 0 \%}$ ) and cerium nitrate hexahydrate (Alfa Aesar, 99.99\%) in the desired stoichiometric proportions were dissolved in nitric acid (68\%). Typically for an expected $2 \mathrm{~g}$ of elposilite compound, the volume of the starting suspension is equal to $100 \mathrm{~mL}$. The initial molar substitution rates of the doping element were fixed at $2 \%$ and $10 \%$ in this work. These two solutions were mixed and heated under argon flow until total evaporation of the solvent. The resulting solid residue was then heat-treated to remove trace of nitrates and crushed in an agate mortar with half of its weigh of $\mathrm{NH}_{4} \mathrm{HF}_{2}$. A final thermal treatment at $650{ }^{\circ} \mathrm{C}$ under argon flow for 1 hour was then performed. This step was repeated once. A white polycrystalline powder was obtained.

Single crystal growth. The Bridgman-Stockbarger method was used to grow single crystals. A $50 \mathrm{~g}$ white powder load was introduced in a platinum crucible. This crucible was then placed in a platinum chamber. A primary vacuum $\left(4 \times 10^{-3}\right.$ mbar) was insured by pumping through the platinum tube of the welded cap heading the assembly, crimping of the tube then sealing through tungsten inert gas welding. This assembly was placed in a vertical furnace with four heating zones at a starting temperature of $1170{ }^{\circ} \mathrm{C}$ (the elpasolite melting point is $1094{ }^{\circ} \mathrm{C}$ ). The furnace was then translated upward at $0.83 \mathrm{~mm} \mathrm{~h}^{-1}$ around the platinum assembly, allowing the slow cooling of the material. Centimetre-sized single crystals with various shapes were obtained. Single crystals issued were labelled LC and HC crystals for the lowest (2\%) and the highest (10\%) targeted concentrations of cerium, respectively.

Luminescence characterization. The photoluminescence properties were analysed using a Horiba Fluorolog 3 spectrofluorimeter. The excitation spectra were corrected for the variation of the incident flux, and the emission spectra were corrected for the transmission of the monochromator and the response of the photomultiplier. The equipment used consisted of a $450 \mathrm{~W}$ xenon lamp, an excitation double monochromator, an emission double monochromator and a thermoelectrically cooled photomultiplier tube. Measurements in temperature and controlled atmospheres were performed in a linkam CCR1000 Microvision Instrument cell coupled with the Horiba optical fibre that was connected to the previously described equipment.

X-ray diffraction analysis. Powder X-ray diffraction patterns were collected on a Philips X'Pert MPD X-ray diffractometer with a Bragg-Brentano geometry using $\mathrm{Cu} \mathrm{K}_{\alpha} 1,2$ radiation $\left(10<2 \theta<130^{\circ}\right.$, with a step size of $0.02^{\circ}$ and a counting time of $30 \mathrm{~s})$. The diffractograms were refined using the Rietveld method with the conventional reliability factors. The Fullprof program package was used. ${ }^{16}$ The unit cell parameters, atomic positions and Debye-Waller factors were refined on the basis of the $F m \overline{3} m$ space group corresponding to the elpasolite structure.

ICP-OES measurements. The inductively Coupled Plasma Optical Emission Spectroscopy (ICP-OES) was performed with a Varian 720ESD apparatus equipped with an argon plasma torch and a CDD detector display. The samples were prepared by their dissolution in chloric acid aqueous solutions.

Magnetic measurements. The magnetic measurements were performed in a static magnetic flux (DC) with a MPMS-5S SQUID (Superconducting Quantum Interference Device) apparatus. The magnetizations were performed between 2 and $100 \mathrm{~K}$ in an applied field ranging in the $0 \mathrm{~T}-5 \mathrm{~T}$ range within a zerofield-cooled process. Data collected at $100 \mathrm{~K}$ were fitted to extract the diamagnetic contribution from the sample holder and $\mathrm{Rb}_{2} \mathrm{KInF}_{6}$ host.

\section{Experimental results}

\section{Structural analyses}

The X-ray data obtained on a crushed LC (targeted concentration equal to $2 \%$ ) and $\mathrm{HC}$ (targeted concentration equal to $10 \%$ ) single crystal illustrate a pure elpasolite phase (Fig. 1).

Fig. $\mathrm{S} 1 \dagger$ shows the platinum crucible after the two crystal growth attempts and the as-grown Ce-doped single crystals.
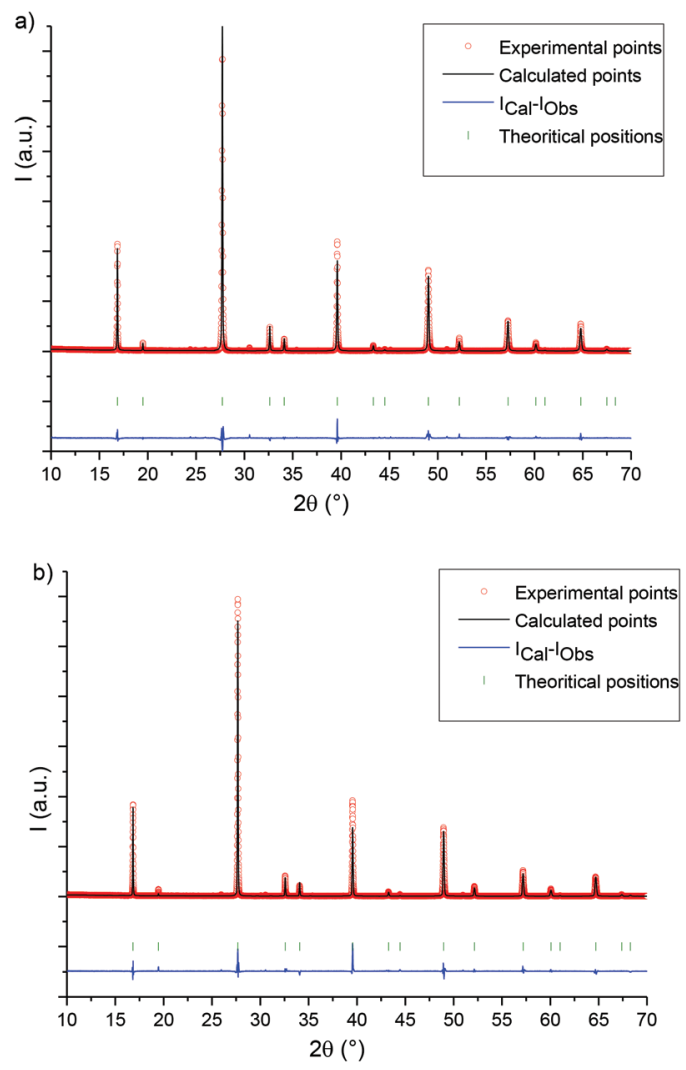

Fig. 1 Rietveld diffraction pattern refinements illustrated on $\mathrm{Rb}_{2} \mathrm{KInF}_{6}$ : $\mathrm{Ce}$ (a) LC crystal (targeted concentration equal to $2 \%$ ) and (b) HC crystal (targeted concentration equal to $10 \%$ ). 
Single crystals are located on the border of the crucible, whereas an opaque zone is visible in the centre (zone inside the dotted line). These solid residues were analysed by X-ray diffraction: polycrystalline mixtures were detected. These polycrystalline mixtures were composed of the $\mathrm{Rb}_{2} \mathrm{KInF}_{6}$ elpasolite phase as the main phase and secondary $\mathrm{KCe}_{2} \mathrm{~F}_{9}$ (JCPDS no. 00025-0632) and $\operatorname{RbIn}_{3} \mathrm{~F}_{10}$ (00-028-0908) phases for the batch with the lowest cerium content and $\mathrm{KCeF}_{4}$ (no. 00-025-0585) and $\mathrm{RbIn}_{3} \mathrm{~F}_{10}$ (no. 00-028-0908) phases for the batch with the highest cerium content. The single crystal parts crushed for the X-ray analyses reported in Fig. $1 \mathrm{a}$ and $\mathrm{b}$ are peripheral colourless single crystal parts.

The evolution of the $a$ cell parameter (see Table 1) of the Ce-doped $\mathrm{Rb}_{2} \mathrm{KInF}_{6}$ compounds confirms the introduction of at least a significant fraction of the cerium ions into the host lattice. Comparison between LC and HC crushed crystal refinements also indicates that the rate of cerium that is really introduced varies as a function of the target doping value. The cation-fluorine distances in the octahedral environment have been modified, especially the increase of the In-F distances

Table 1 Cell parameter refinements of $\mathrm{Rb}_{2} \mathrm{KlnF}_{6}$ compounds. Fluorine anions were positioned on the $96 \mathrm{j}$ site

\begin{tabular}{|c|c|c|c|c|c|c|}
\hline & \multirow[b]{2}{*}{$a(\AA)$} & \multirow[b]{2}{*}{$d_{\text {In-F }}(\AA)$} & \multirow[b]{2}{*}{$d_{\mathrm{K}-\mathrm{F}}(\AA)$} & \multicolumn{3}{|c|}{ Reliability factors } \\
\hline & & & & $R_{\mathrm{p}}$ & $\mathrm{Chi}^{2}$ & $\begin{array}{l}\text { Bragg } \\
R \text {-factor }\end{array}$ \\
\hline Non-doped ${ }^{13}$ & $909156(1)$ & $2038(4)$ & $2508(4)$ & - & - & $5.95^{a}$ \\
\hline LC crystal & $90933(5)$ & 2063(7) & $2559(2)$ & 17.8 & 2,72 & 4,84 \\
\hline HC crystal & $91023(5)$ & 2096(1) & $2531(2)$ & 9.24 & 3,72 & 7,59 \\
\hline
\end{tabular}

${ }^{a}$ Bragg integral discrepancy factor. versus the cerium target doping value. This last observation shows the preferential incorporation of the cerium ions into the indium Wyckoff positions. The small proportion of cerium located on the cuboctahedron site detected in previous optical characterizations does not impact these structural data. ${ }^{15}$

As the X-Ray analysis performed after crystal growth indicates a segregation of cerium in the $\mathrm{KCeF}_{4}, \mathrm{KCe}_{2} \mathrm{~F}_{9}$ and probably in the $\mathrm{RbIn}_{3} \mathrm{~F}_{10}$ phases, chemical analyses were run to determine the cerium content within the single crystals. The results obtained for LC and HC single crystals are reported in Table 2. Cerium, indium, potassium and rubidium elements were analysed, and the titration was repeated twice (i.e., two different solutions were prepared).

As indicated in Table 2, the experimental and theoretical titrations of indium, potassium and rubidium elements are equivalent, but the real relative content of cerium is $50 \%$ less important than the target content for HC Ce-doped crystals: the average calculated molar percentage of cerium obtained on two titrations is equal to $4.6 \%$ instead of $10 \mathrm{~mol} \%$. The titration experiments were performed on the $2 \mathrm{~mol} \%$ Ce-doped compound, but the very low cerium concentration of the prepared solutions ([Ce $<1 \%]$ ) in comparison with the other element concentrations ( $\mathrm{Rb}, \mathrm{K}$, In) leads to divergent values for the cerium content. Considering these data, one can confirm that the crystal growth induces a loss of about half of the rare earth ions due the purification of the elpasolite host in favour of Ce-rich secondary phases.

\section{Optical properties}

The luminescence of the $\mathrm{Rb}_{2} \mathrm{KInF}_{6}: \mathrm{Ce}^{3+}$ material is due mainly to the presence of trivalent cerium, as described in our previous published study on a Ce-doped single crystal. ${ }^{7,15}$ The

Table $2 \mathrm{ICP}$ results on $\mathrm{LC}$ and $\mathrm{HC}$ Ce-doped $\mathrm{Rb}_{2} \mathrm{KInF}_{6}$ single crystal

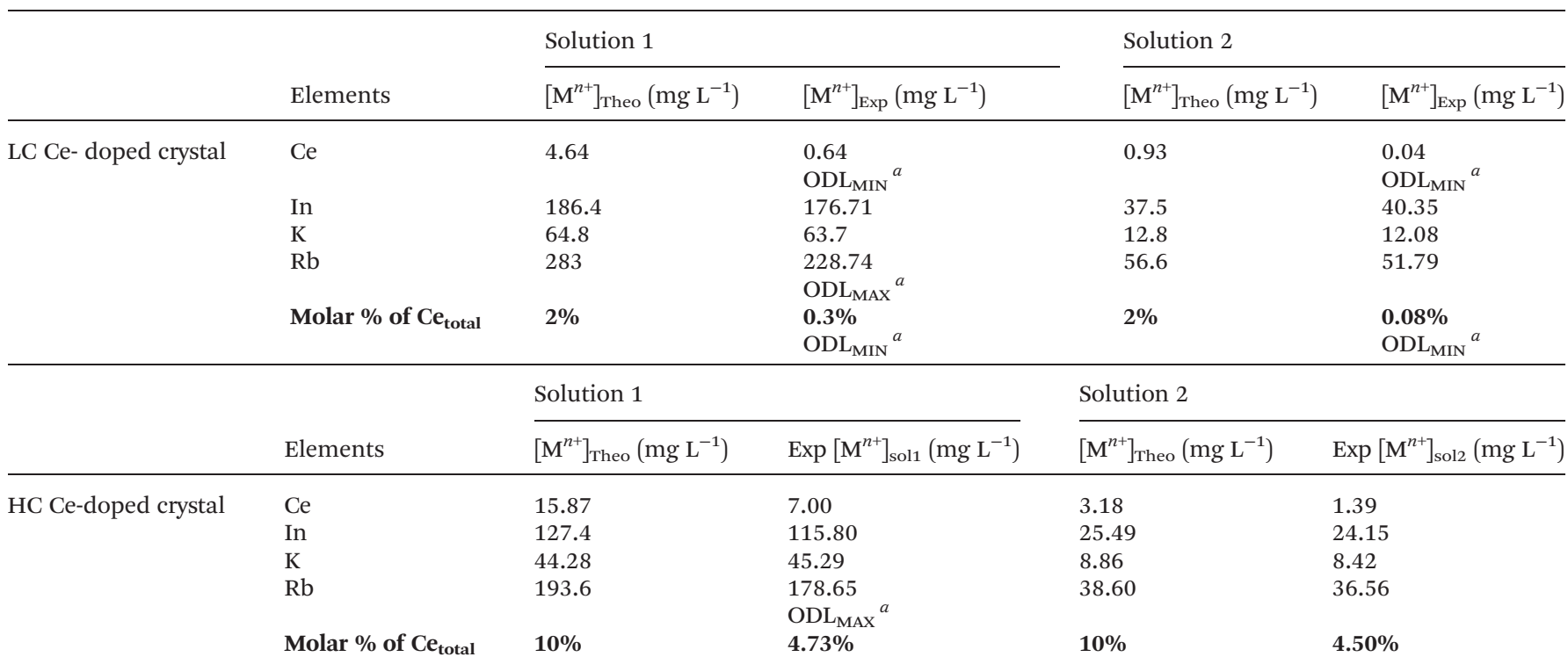

${ }^{a}$ ODL: Over Detection Limit. 


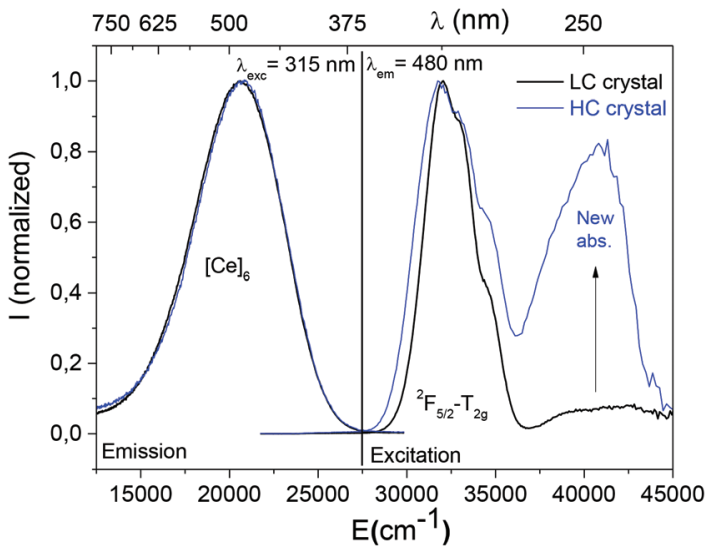

Fig. 2 Room temperature luminescence spectra of $\mathrm{LC}([\mathrm{Ce}]<1 \%)$ (black curves) and $\mathrm{HC}\left([\mathrm{Ce}]<4.6 \%\right.$ ) (blue curves) Ce-doped $\mathrm{Rb}_{2} \mathrm{KInF}_{6}$ single crystal $\left(\lambda_{\mathrm{exc}}=315 \mathrm{~nm}, \lambda_{\mathrm{em}}=480 \mathrm{~nm}\right)$.

integration of cerium cations occurs in both the octahedral (indium site) and cuboctahedral (rubidium site) polyhedra of the elpasolite structure. The first one is maximum at around $480 \mathrm{~nm}\left(20830 \mathrm{~cm}^{-1}\right)$ whereas the second band is much weaker in intensity and points in the UV range at $340 \mathrm{~nm}$ $\left(29410 \mathrm{~cm}^{-1}\right)$. As the cerium content of the HC crystal is much higher than the previous LC studied crystal, local disorders are expected and it is worthy to re-investigate the impact of the substitution within the host, on the optical properties.

The room temperature excitation and emission graphs of the LC $([\mathrm{Ce}]<1 \%$ molar $)$ and $\mathrm{HC}([\mathrm{Ce}]=4.6 \%$ molar $)$ ceriumdoped crystals are illustrated in Fig. 2. The description of the emission properties will be focussed on the visible range and the UV contribution of cerium ions in the cuboctahedron site will not be reported as it is not affected by the irradiation. ${ }^{7,15}$

The detected visible emission centred at $480 \mathrm{~nm}$ $\left(20830 \mathrm{~cm}^{-1}\right)$ and observed with an excitation at $315 \mathrm{~nm}$ $\left(31745 \mathrm{~cm}^{-1}\right)$ has been attributed to the location of trivalent cerium in the six-fold coordinated site $\left([\mathrm{Ce}]_{6}\right)$. The excitation curves associated with the emission at $480 \mathrm{~nm}\left(20830 \mathrm{~cm}^{-1}\right)$ show the splitting of the different $5 \mathrm{~d} \mathrm{~T}_{2 \mathrm{~g}}$ levels of trivalent cerium between $280 \mathrm{~nm}$ and $340 \mathrm{~nm}$, which corresponds to the contribution of trivalent cerium to the octahedral environment. Surprisingly even if the shape of the emission band is equivalent for both crystals, the corresponding excitation curves revealed a new contribution at $255 \mathrm{~nm}\left(39215 \mathrm{~cm}^{-1}\right)$ of the HC crystal. This additional absorption band reflects the existence of a new emitting centre.

The visible emission observed under $255 \mathrm{~nm}\left(39215 \mathrm{~cm}^{-1}\right)$ excitation beam, is shown in Fig. 3. An intense green contribution labelled [?] appears as a large band pointing at $510 \mathrm{~nm}$ $\left(19600 \mathrm{~cm}^{-1}\right)$ on the HC crystal emission curve whereas no signal is detected on the LC material. Because of the overlap between the [?] contribution and the $480 \mathrm{~nm}$ emission of cerium ions in the indium octahedral site, absorption of the $5 \mathrm{~d} \mathrm{~T}_{2 \mathrm{~g}}$ levels are also visible in excitation spectra performed at $\lambda_{\mathrm{em}}$ equal to $510 \mathrm{~nm}$.

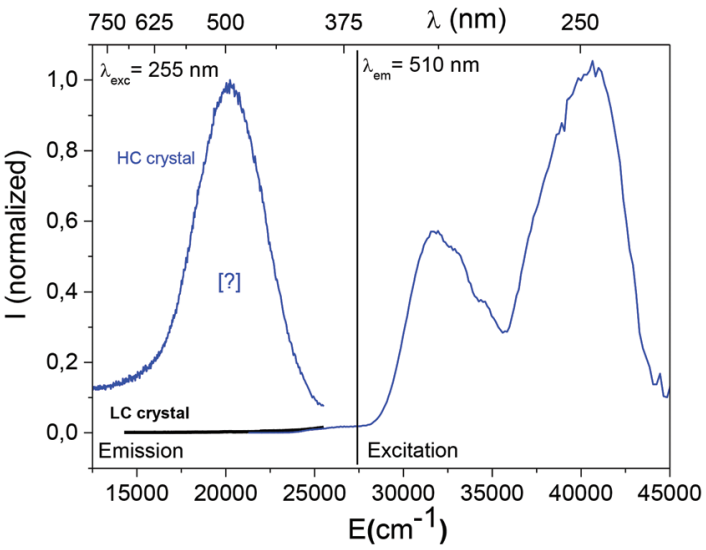

Fig. 3 Emission spectrum of a $<1 \%$ (LC) Ce-doped $\mathrm{Rb}_{2} \mathrm{KInF}_{6}$ single crystal (black curve) under $255 \mathrm{~nm}$ excitation beam and luminescence spectra $4.6 \%(\mathrm{HC}) \mathrm{Ce}$-doped $\mathrm{Rb}_{2} \mathrm{KInF}_{6}$ single crystal (blue curves) $\left(\lambda_{\text {exc }}=\right.$ $\left.255 \mathrm{~nm}, \lambda_{\mathrm{em}}=510 \mathrm{~nm}\right)$.

The initial raw powder prepared for the HC crystal growth was controlled to check its spectral fingerprint under the same irradiation conditions. But, no emission was detected in the visible range under a $255 \mathrm{~nm}$ excitation beam. The crystal growth induces local modifications that stabilize a new emitting centre.

To better understand the origin of this new luminescent centre, comparison with visible optical properties obtained on a material after an irradiation step of 2 hours at $315 \mathrm{~nm}$ was performed. As this material is sensitive to UV irradiation, when it is exposed to a $315 \mathrm{~nm}$ excitation beam (irradiation process), the intensity of the emission peak associated with the six-fold coordinated cerium ions (located on the indium Wyckoff positions) decreases, whereas a red emission appears at $650 \mathrm{~nm}$ under excitation at $255 \mathrm{~nm}$ following the equilibrium $2 \mathrm{Ce}^{3+}+\mathrm{In}^{3+} \rightarrow 2 \mathrm{Ce}^{4+}+\mathrm{In}^{+}$. This last orange emission has already been shown to be due to monovalent $\mathrm{In}^{+}$cations in the octahedral site of the elpasolite structure. ${ }^{7}$

The excitation and emission graphs performed on the irradiated HC crystal are shown in Fig. 4.

An excitation at $255 \mathrm{~nm}$ reveals two components in the emission spectrum: the new [?] contribution peaking at $510 \mathrm{~nm}$, which seems not to be affected by the temperature, and the monovalent indium contribution composed of the $\mathrm{A}_{T}$ band $\left(400 \mathrm{~nm} / 25000 \mathrm{~cm}^{-1}\right)$ detected only at low temperatures and the $A_{X}$ band (at approximately $650 \mathrm{~nm} / 15300 \mathrm{~cm}^{-1}$ ). ${ }^{15,17,18}$ The excitation spectra that correspond to the monovalent indium absorption $\left(\lambda_{\mathrm{em}}=400 \mathrm{~nm}, 650 \mathrm{~nm}\right)$ are formed by a unique band at $255 \mathrm{~nm}\left(39215 \mathrm{~cm}^{-1}\right)$ that peaks in the same energy range as the range of the new emitting centre.

\section{Magnetism}

To determine the ratio of $\mathrm{Ce}^{3+} / \mathrm{Ce}^{4+}$ ions in the crystals, magnetization versus applied magnetic field measurements were performed. Plotted in Fig. $\mathrm{S} 2 \dagger$ is the magnetization corrected from the sample holder and the host diamagnetic signal versus 

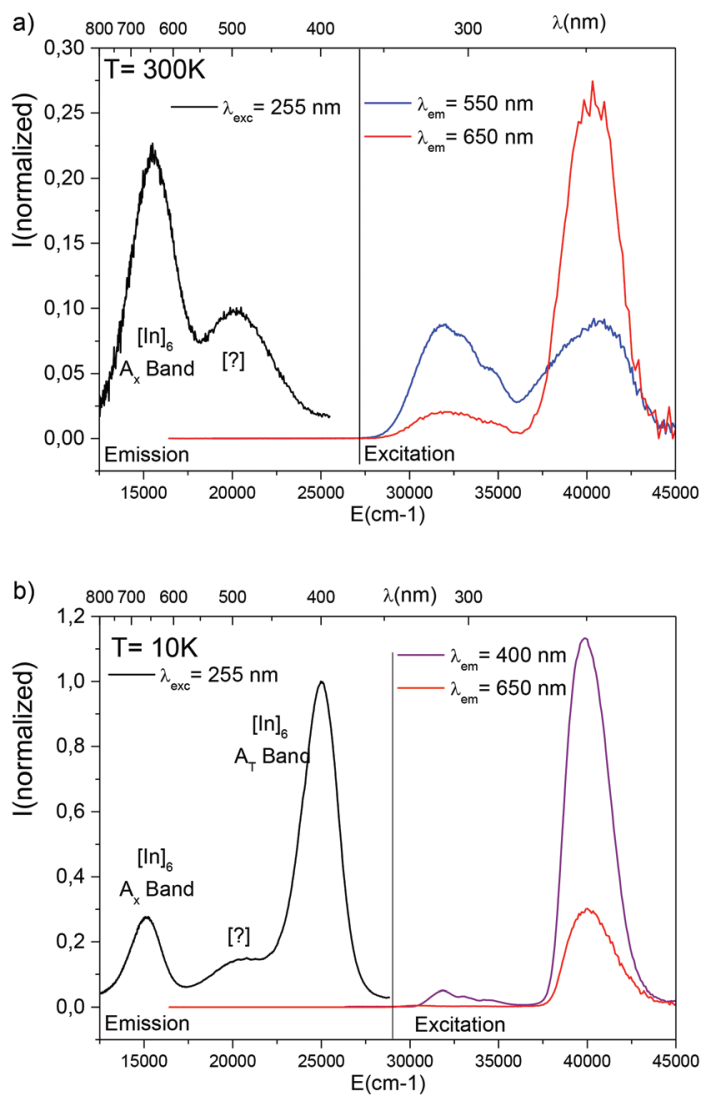

Fig. 4 Room (a) and low temperature (10 K) (b) luminescence spectra after a 2-hour irradiation at $315 \mathrm{~nm}$ showing the contribution of the indium ions reduced under the UV beam on the $4.6 \%$ cerium content $\mathrm{Rb}_{2} \mathrm{KInF}_{6}$ crushed crystal ( $\left.\mathrm{HC}\right)$.

the $\mathrm{H} / \mathrm{T}$ ratio for data collected at $2 \mathrm{~K}, 3 \mathrm{~K}$ and $5 \mathrm{~K}$ on the crushed LC and HC single crystals. The as-highlighted paramagnetic signal is due only to $\mathrm{Ce}^{3+}$ in relation to its $4 \mathrm{f}^{1}$ configuration $(J=5 / 2)$.

To analyse the $\mathrm{H} / \mathrm{T}$ ratio dependence of the magnetic signal more deeply, the collected data were fitted by the Brillouin function. The magnetization is given by $M=N_{\mathrm{A}} g J \mu_{\mathrm{B}} B_{J}(x)$ with $N_{\mathrm{A}}$ : Avogadro's number, $g$ : the effective $g$-value, $J$ : the total angular moment, $\mu_{\mathrm{B}}$ : the Bohr magneton, $x$ : the $g J \mu_{\mathrm{B}} H_{\mathrm{z}} / k_{\mathrm{B}} T$ ratio (with $H_{\mathrm{z}}$ the applied magnetic field and $T$ the temperature) and $B_{J}(x)$ : the Brillouin function defined by:

$$
B_{J}(x)=\frac{2 J+1}{2 J} \operatorname{coth}\left(\frac{(2 J+1) x}{2 J}\right)-\frac{1}{2 J} \operatorname{coth}\left(\frac{x}{2 J}\right)
$$

The fitted parameter, i.e., the $g$-value, and the percentage of trivalent cerium contributed to the signal are given in Table 3. The angular momentum was fixed at 5/2.

From the ICP results, a low cerium content is expected, and our results showed that most of the cerium is diamagnetic with an oxidation state of $\mathrm{Ce}^{4+}$. Considering the HC Ce-doped compound, a total $\mathrm{Ce}^{3+}$ ion concentration of $0.348 \%$ was found in the single crystals, which is far from the total cerium concentration rate of $4.6 \%$ determined by chemical analysis.
Table 3 Fitted parameters ( $g$-value and Ce(III) concentration) for the two concentrations

\begin{tabular}{lll}
\hline Sample & Ce $(\ll 1 \%)$ crystal growth & Ce $4.6 \%$ crystal growth \\
\hline$g$ & 1.36 & 0.78 \\
$\%$ Ce(III) & 0.081 & 0.348
\end{tabular}

Only $7.5 \%$ of the cerium is stabilized at the oxidation number III. The trivalent cerium concentration for the LC Ce-doped material was evaluated at $0.081 \%$, i.e., approximately four times lower than for the HC Ce-doped samples. The discrepancies shown by the $g$-values are interesting. For a free $\mathrm{Ce}^{3+}$ ion, a value of $6 / 7$ is expected for the Lande factor where the $g$-value can differ from $g_{J}$ due to a crystal field effect that splits the ground state into doublets. A $g$-value of 1.16 was obtained for $\mathrm{Ce}^{3+}$-doped $\mathrm{Sr}_{0.61} \mathrm{Sr}_{0.39} \mathrm{Nb}_{2} \mathrm{O}_{6}$ showing a $3 \mathrm{D}$ network of $\mathrm{NbO}_{6}$ octahedra linked by their corners forming alternating five-and four-membered rings. ${ }^{19}$

\section{Discussion}

All of these characterizations converge towards a lower rate of occurrence of the cerium element in the crushed crystals in comparison with the target rate (i.e., the initial rate introduced in the starting solution used as the co-precipitation medium). Indeed, the crystal growth purifies the elpasolite host lattice by favouring the spatial segregation of the secondary phases as $\mathrm{KCeF}_{4}, \mathrm{KCe}_{2} \mathrm{~F}_{9}$ and $\mathrm{RbIn}_{3} \mathrm{~F}_{10}$. Unfortunately, these separated secondary phases are cerium-rich phases. The magnetism measurements indicate that the main parts of the cerium ions inside the crystals are not magnetic: they are stabilized mainly with the +IV oxidation number $(92.5 \%$ in the HC Ce-doped crystals).

The crystal growth step seems to favour the oxidation of the doping element. In previous work, ${ }^{15}$ DFT calculation was used to show that the cerium ions located in the indium site are under an oxidative stress, which was not confirmed at this time, by magnetic measurements. To compensate for the excess of positive charge, the structure may induce punctual defects such as substitution of $\mathrm{O}^{2-}$ anions on $\mathrm{F}^{-}$sites, cationic vacancy on the alkali sites or reduction of neighbour cations such as indium. Indeed, in cerium-doped fluorine compounds such as $\mathrm{SrAlF}_{5}$ or $\mathrm{LaF}_{3}$, red-shifted cerium emission bands have been reported. The origins of these cerium emission bands are due to perturbed cerium ions. These specific sites are formed by a fluorine defect that is charge-compensated by the presence of oxygen anions in the matrix. In this case, the corresponding excitation spectra are composed of several bands, the first band being shifted to the lower energy. ${ }^{20,21}$ The second hypothesis concerns the alkali vacancies. They are not predominant, as the chemical analysis results did not indicate any deficit in potassium or rubidium elements. Finally, the possibility that indium ions are initially in the monovalent 
oxidation state in the single crystals is considered. The existence of such a centre may have a direct impact on the luminescence properties. Considering all of the data exposed, we can discard the oxygen-type defect as the excitation band peaks at $255 \mathrm{~nm}$. The chemical analyses, the large Stokes shift calculated (approximately $20000 \mathrm{~cm}^{-1}$ ) between the excitation and absorption band of the blue-green new emission, the facts that it is detected at room temperature and almost not affected by the temperature and irradiation exposure seem to eliminate punctual defects as alkali vacancies in the array as the origin of the phenomenon. The more valid hypothesis is based on the reduction of trivalent indium. Contrary to what is observed for the indium ions in the octahedral environment, no indication of the splitting of the excited levels in $A_{T}$ and $A_{X}$ bands is detected on the excitation or emission graphs performed at low temperature, implying that the reduced centres that appear after crystal growth are not in the same octahedral environment as the monovalent indium ions reduced after the $315 \mathrm{~nm}$ irradiation step. Because the crystal growth is a lengthy process, one can suggest that crystal growth allows the mobility of cations in the structure and stabilizes the monovalent indium element in the biggest host site of the structure (i.e., the cuboctahedral site). No splitting of the excited states is expected in this symmetry. Because the proximity to the cerium element is different from the proximity of the indium ions localized in their expected crystallographic position, no electron transfer is optically initiated between these new reduced cations and the rare earth elements. The stability of these centres under irradiation should therefore be explained. The lack of additional emission in the LC cerium doped single crystals can be justified by the concentration of cerium $([\ll 1 \%])$ within the grown materials that is too low. As a reminder, magnetic measurements have indicated a value of $0.081 \%$ of trivalent cerium.

Note: considering the allowed $5 \mathrm{~s}-5 \mathrm{p}$ transitions of monovalent indium, a short lifetime of this new luminescent centre is expected but the recording of this emission cannot be performed as this new emitting centre and $[\mathrm{Ce}]_{6}$ ions ranges in the same energy domain, the optical instability of the latter preventing a correct measurement.

\section{Cyclability test}

Cyclability test on crushed single crystals. Both of the Cedoped prepared samples express photo-chromo-luminescence properties. The redox phenomenon is reversible, and the initial emission properties can be restored by exposure at $255 \mathrm{~nm}$. To test the aging properties of this phenomenon, cycling studies have been performed on crushed crystals.

The following irradiation cycles were applied to the two HC $\left(0.348 \% \mathrm{Ce}^{3+}\right.$ content) and LC $\left(0.081 \% \mathrm{Ce}^{3+}\right.$ content $)$ doped single crystals. The crushed single crystals were successively exposed for 1 hour at $315 \mathrm{~nm}$, then 1 hour at $255 \mathrm{~nm}$, and so on for six cycles. The raw crystals (not irradiated) are associated to the $t_{0}$ emission curve (and the cycle noted 0 ); after 1 hour irradiation at $315 \mathrm{~nm}$, the samples are associated to the $t_{1}$ curve (cycle 1); the samples obtained after the 1st cycle of irradiation were newly irradiated 1 hour at $255 \mathrm{~nm}\left(\mathrm{t}_{2}\right.$ curve, cycle 2), and so on for six cycles. Whatever the irradiation cycle, the emission spectra were recorded at a fixed excitation wavelength of $255 \mathrm{~nm}$.

Fig. 5 illustrates the emission properties with excitation at $255 \mathrm{~nm}$ and the corresponding variation of the integrated area as a function of the cycling step. The black emission curve illustrates the optical response of the raw crystals under a $255 \mathrm{~nm}\left(\mathrm{t}_{0}\right)$. The red curve was performed under the same experimental excitation $\left(\lambda_{\text {exc }}=255 \mathrm{~nm}\right)$ after a 1 hour irradiation at $315 \mathrm{~nm}$ of the sample $\left(t_{1}\right)$. Finally, the blue curve emission $\left(t_{2}\right)$ illustrates the re-oxydation of the monovalent indium associated with the disappearance of the orange band. The global integrated area of the emission curves were calculated in the $400 \mathrm{~nm}-800 \mathrm{~nm}$ range. The corresponding values are reported as black dot in Fig. $5 \mathrm{~b}$ and $5 d$, at the end of each irradiation step. Photographs of the crushed HC and LC crushed Ce-doped crystals under a 255 excitation beam are shown in parallel for each step.

The "on-off" phenomenon of the $\operatorname{In}^{+}$generated under irradiation is mostly reversible. The lowest cerium content single crystal (LC) shows an orange luminescence that is fully erased by the reverse irradiation cycle ( 1 hour irradiation at $255 \mathrm{~nm}$ performed after the 1 hour irradiation at $315 \mathrm{~nm}$ ). The calculated trichromatic coordinates are $x=0.462$ and $y=0.357$. The highest cerium content crystal (HC) alternatively shows a bluish-green $(0.2238, Y=0.3639)$ and a yellowish-orange $(x=$ $0.3549, y=0.3826$ ) luminescence due to the contribution of the new emitting centre. A "nice switch" between two visible colours is therefore obtained for the HC Ce-doped crystals. For both materials, the proportion of neighbour trivalent cerium
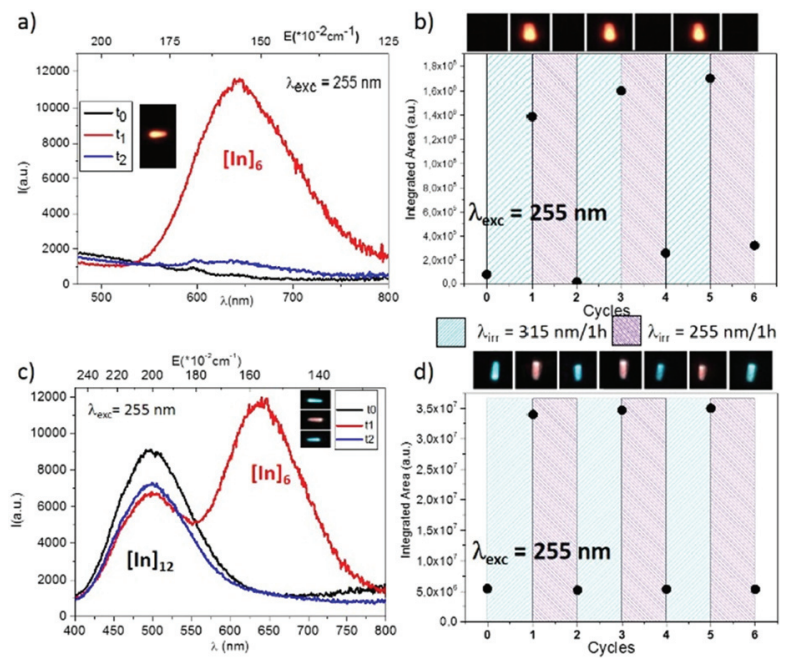

Fig. 5 Ce-doped $\mathrm{Rb}_{2} \mathrm{KInF}_{6}$ emission characterization. (a) $\mathrm{Ce}^{3+} 0.081 \%$ (LC crystal) emission spectra at different cycle steps under $\lambda_{\text {exc }}=$ $255 \mathrm{~nm}$, (b) integrated area of emission curves with powder photographs of the LC crushed crystal under $\lambda_{\text {exc }}=255 \mathrm{~nm}$, (c) $\mathrm{Ce}^{3+} 0.348 \%$ (HC crystal) emission spectra at different cycle steps under $\lambda_{\text {exc }}=255 \mathrm{~nm}$, (d) integrated area of emission curves with powder photographs of the $\mathrm{HC}$ crushed crystal under $\lambda_{\text {exc }}=255 \mathrm{~nm}$. 


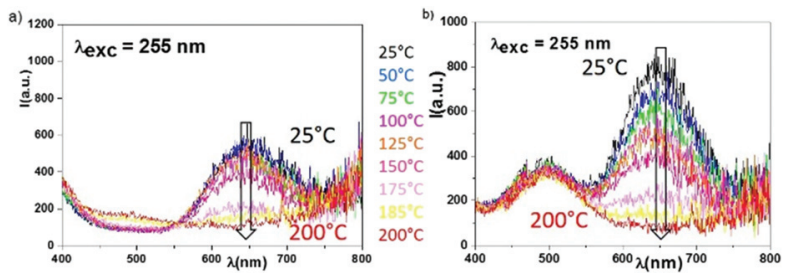

Fig. 6 Room temperature emission graphs under a $255 \mathrm{~nm}$ excitation beam obtained after a 2-hour irradiation at $315 \mathrm{~nm}$. The irradiated crushed crystals were successively heated from $25^{\circ} \mathrm{C}$ to $200{ }^{\circ} \mathrm{C}$ under a reductive atmosphere of $\mathrm{Ar} / \mathrm{H}_{2} 10 \%$. (a) $0.081 \%$ of $\mathrm{Ce}^{3+}$ content (LC) and (b) $0.348 \%$ of $\mathrm{Ce}^{3+}$ content $(\mathrm{HC})$.

and indium cations is sufficient to make possible the $2 \mathrm{Ce}^{3+}+$ $\mathrm{In}^{3+} \leftrightarrow 2 \mathrm{Ce}^{4+}+\mathrm{In}^{+}$redox process.

As a complement, thermal tests (10 minutes of heat treatment at each temperature and cooling to room temperature between two thermal steps) were performed to check the effect of temperature on the reversible redox process. Samples irradiated for 2 hours at $315 \mathrm{~nm}$ were prepared to stabilize $\mathrm{In}^{+}$ions. These samples were heated successively at different temperatures between $25{ }^{\circ} \mathrm{C}$ and $200{ }^{\circ} \mathrm{C}$, and the system was cooled between each of the thermal steps to record the room temperature emissions observed under a $255 \mathrm{~nm}$ excitation beam.

Results are illustrated in Fig. 6. As shown, a total erasure of the monovalent indium luminescence generated (in an octahedral environment) is obtained under a heat treatment at $200{ }^{\circ} \mathrm{C}$.

In addition, room temperature kinetic tests were also performed to follow the decrease of the $\mathrm{Ce}^{3+}$ emission under a $315 \mathrm{~nm}$ irradiation beam under argon, nitrogen, oxygen and argon/hydrogen atmospheres. No significant difference was observed, which confirms that the redox phenomenon is totally independent from the atmosphere (see ESI, Fig. S3 $\dagger$ ).

"Printing" test on single crystals. Such sensibility for UV radiation makes this material a good candidate for application in dosimetry by following the kinetics of the irradiation. For both cerium content crystals, even if the redox process begins at the first step of the irradiation, the decreasing kinetic curves proving the reoxydation of monovalent indium, reach a plateau for irradiation time superior to 6000 seconds (see ESI, Fig. $\mathrm{S} 4 \dagger$ ). As centimetric single crystals were grown, their large surface can be used to reveal the colorimetric contrast of selective irradiated zone.

Three types of areas are presented in Fig. 7: white areas were not exposed to UV radiation (reference), the red areas were exposed to irradiation at $315 \mathrm{~nm}$, and the blue areas were irradiated successively at $315 \mathrm{~nm}$ and $255 \mathrm{~nm}$. These results show that the pattern can be printed and erased, and considering the cyclability test results, this cycle can be repeated at least three times. Moreover, even if the irradiated materials are stored in air, sheltered from UV irradiation, the exposed zones are very stable, as the control of a twenty-year irradiated single crystal still reveals the orange emission of monovalent indium.
"Printing" test on single crystals

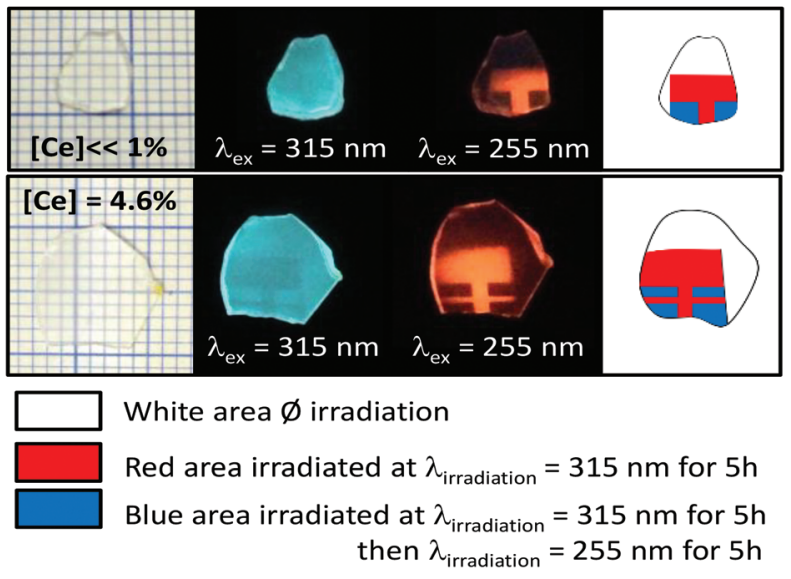

Fig. 7 Printing tests performed on single crystals. Photographs of $\mathrm{Ce}-$ doped $\mathrm{Rb}_{2} \mathrm{KInF}_{6}$ single crystals after several irradiation processes.

\section{Conclusions}

X-Ray diffraction, chemical analyses and magnetism confirmed the cerium doping of the $\mathrm{Rb}_{2} \mathrm{KInF}_{6}$ single crystal grown through a Bridgman technique. Surprisingly, total amounts of cerium within the hosts were much lower than the targeted concentrations of $2 \%$ and $10 \%$. Among the rare earth ions introduced into the lattice, main part is stabilized in the IV oxidation state, leading to $0.081 \%$ and $0.348 \%$ trivalent ceriumdoped crystals. For the highest concentrated sample (HC crystal), the charge compensation required by the stabilization of a higher initial proportion of tetravalent cerium ions is ensured by the reduction of indium cations to the monovalent oxidation state, leading the observation of a new bluish-green emission which is not observed on the LC crystal. A location of monovalent indium ions in the cuboctahedral site is proposed.

The reversible $2 \mathrm{Ce}^{3+}+\mathrm{In}^{3+} \leftrightarrow 2 \mathrm{Ce}^{4+}+\mathrm{In}^{+}$process was studied as a function of the temperature and under several atmospheres. A $200{ }^{\circ} \mathrm{C}$ heat treatment seems to be sufficient to re-oxidize the $\mathrm{In}^{+}$UV-generated cations to the trivalent oxidation state. As expected, the redox process appears to be independent of the atmosphere.

Finally, for the first time, cycling and printing tests were performed. A perfect "on-off switch" is visible for the LC Cedoped material, whereas a nice "bluish-green $\rightarrow$ orange switch" is observable for the HC Ce-doped material. Results are reproducible over at least four cycles, which confirms that these materials are in good agreement with UV dosimetry devices.

\section{Acknowledgements}

Lucile Cornu was the holder of the doctoral fellowship supported by the University of Bordeaux. The authors thank Dr 
Alain Garcia for fruitful discussion. The authors thank the CNRS and the Aquitaine region. This study was carried out with financial support from the French State, managed by the French National Research Agency (ANR) in the frame of a nonthematic program (ANR-2010-BLANC-0820) and in the frame of "the Investments for the future" Program IdEx Bordeaux LAPHIA (ANR-10-IDEX-03-02).

\section{Notes and references}

1 P. A. Tanner, Top. Curr. Chem., 2004, 167-278, 241(Transition Metal and Rare Earth Compounds III).

2 (a) S. S. Pedro, L. P. Sosman, R. B. Barthem, J. C. G. Tedesco and H. N. Bordallo, J. Lumin., 2013, 134, 100; (b) L. J. Andrews and S. M. Hitelman, Ettore Majorana Int. Sci. Ser.: Phys. Sci., 1987, 30, 515.

3 M. Laroche, M. Bettinelli, S. Girard and R. Moncorgé, Chem. Phys. Lett., 1999, 311(3-4), 167.

4 H. Ramanantoanina, W. Urland, F. Cimpoesu and C. Daul, Phys. Chem. Chem. Phys., 2013, 15, 13902.

5 H. Ramanantoanina, W. Urland, F. Cimpoesu and C. Daul, Phys. Chem. Chem. Phys., 2013, 15(33), 13902.

6 J. Glodo, E. van Loef, R. Hawrami, W. M. Higgins, A. Churilov, U. Shirwadkar and K. S. Shah, IEEE Trans. Nucl. Sci., 2011, 58, 333.

7 J. P. Chaminade, A. Garcia, T. Gaewdang, M. Pouchard, J. Grannec and B. Jacquier, Radiat. Eff. Defects Solids., 1995, $135,137$.
8 P. Mesnard, PhD Thesis, Université de Bordeaux 1, 1997.

9 H. Guengard, PhD Thesis, Université Bordeaux 1, 1994.

10 Y. Xu, S. Carlson, A. Sjodin and R. Norrestam, J. Solid State Chem., 2000, 150, 399.

11 B. F. Aull and H. P. Jenssen, Phys. Rev. B: Condens. Matter, 1986, 34, 6640.

12 A. Tressaud, J. Darriet, P. Lagassie, J. Grannec and P. Hagenmuller, Mater. Res. Bull., 1984, 19, 983.

13 W. J. Cross and W. J. Hillebrand, Z. Krystallogr. Mineral., 1887, 12, 495.

14 K. S. Aleksandrov, S. V. Misyul, M. S. Molokeev and V. N. Voronov, Phys. Solid State, 2009, 51, 2505.

15 L. Cornu, M. Gaudon, P. Veber, A. Villesuzanne, S. Pechev, A. Garcia and V. Jubera, Chem. - Eur. J., 2015, 21, 5242.

16 J. Rodriguez-Carvajal, Commission on Powder Diffraction (IUCr) Newsletter, 2001.

17 M. A. Buñuel, B. Moine, B. Jacquier, A. Garcia and J. P. Chaminade, J. Appl. Phys., 1999, 86, 5045.

18 A. Fukuda, Phys. Rev. B: Solid State, 1970, 3(1), 4161.

19 G. Weckwerth, J. Schefer, M. Imlau, M. Wöhlecke, R. Pankrath, D. Schaniel and T. Woike, Phys. Rev. B: Condens. Matter, 2004, 7, 144410.

20 S. I. Omelkov, M. G. Brik, M. Kirm, V. A. Pustovarov, V. Kiisk, I. Sildos, S. Lange, S. I. Lobanov and L. I. Isaenko, J. Phys.: Condens. Matter, 2011, 23, 105501.

21 A. J. Woitowicz, M. Balcerzyk, E. Berman and A. Lempicki, Phys. Rev. B: Condens. Matter, 1994, 49, 14880. 